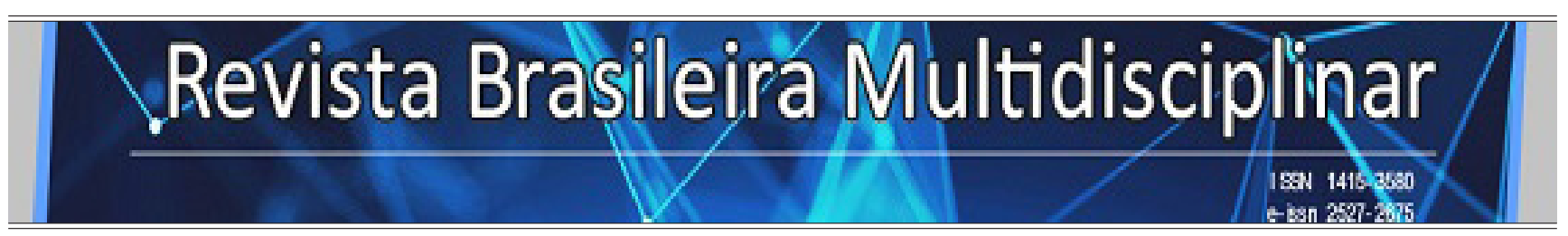

http://revistarebram.com/index.php/revistauniara

\title{
ESTIMATIVA DE TEMPERATURA BASE E FILOCRONO EM QUINOA
}

\author{
Janete Denardi Munareto*; Sandro Petter Medeiros*; Ubirajara Russi Nunes ${ }^{\star *}$; Janine Farias Menegaes ${ }^{\star * *}$. \\ * Mestrado e Doutorado em Agronomia pela Universidade Federal de Santa Maria-RS. \\ ** Doutor, Professor do Departamento de Fitotecnia, Universidade Federal de Santa Maria-RS UFSM. \\ *** Engenharia da Produção Universidade Federal de São Carlos- UFSCAR.
}

*Autor para correspondência e-mail: jdmunareto@gmail.com

\section{Palavras-chave}

Chenopodium Quinoa Aparecimento de Folhas Temperatura do Ar Desenvolvimento Vegetal

\section{KEYWORDS}

Chenopodium Quinoa

Appearance of Leaves

Air Temperature

Plant Development

\begin{abstract}
RESUMO
Os modelos de simulação do desenvolvimento vegetal utilizam o intervalo de tempo entre a emissão de duas folhas consecutivas na haste principal para a alocação do aparato foliar. Esse intervalo é denominado de filocrono e representa a soma térmica acumulada a partir da temperatura base. Com o propósito de aportar informações agronômicas pertinentes às exigências térmicas da cultura da quinoa (Chenopodium quinoa Willd.), o objetivo deste trabalho foi estimar a temperatura base (Tb) e determinar o filocrono do genótipo Q13-31. O experimento foi realizado em Santa Maria, RS, em datas de semeadura: 01/12/2016, 19/01/2017, 01/04/2017, 01/05/2017, 07/10/2017, 09/11/2017, $07 / 12 / 2017$ e 02/01/2018. As plantas foram cultivadas em canteiros, no delineamento de blocos ao acaso, sendo as parcelas constituídas por quatro linhas de um metro em três repetições. A soma térmica diária foi calculada a partir da data da emissão do primeiro ao quinto par de folhas e, o filocrono foi estimado pelo inverso do coeficiente angular da regressão entre número de folhas e soma térmica acumulada. A contagem do número de folhas foi realizada, a cada três dias, até a emissão do quinto par de folhas na haste principal e a temperatura base foi estimada usando o método da menor variabilidade e pela combinação dos métodos de desenvolvimento relativo e de regressão linear. Para o genótipo Q13-31de quinoa, o valor do filocrono é $28^{\circ} \mathrm{C}$ dia folha- 1 enquanto que a temperatura base $(\mathrm{Tb})$ para a emissão de folhas é de $7^{\circ} \mathrm{C}$.
\end{abstract}

\section{ESTIMATE BASE TEMPERATURE AND PHYLLOCHRON IN QUINOA}

The simulation models of plant development use the time interval between the emission of two consecutive leaves on the main stem for the allocation of the leaf apparatus. This interval is called a phyllochron and represents the thermal sum accumulated from the base temperature. With the purpose of providing agronomic information pertinent to the thermal requirements of the quinoa (Chenopodium quinoa Willd.) Culture, the aim of this work was to estimate the base temperature $\mathrm{Tb}$ ) and to determine the phylochron of the Q13-31 genotype. The experiment was carried out in Santa Maria, RS, on sowing dates: 12/1/2016, 1/19/2017, 4/1/2017, 5/1/2017, 10/7/2017, 11/09/2017, $07 / 12 / 2017$ and $01 / 02 / 2018$. The plants were grown in flower beds, in a randomized block design, with plots consisting of four one-meter lines in three replications. The daily thermal sum was calculated from the date of issue of the first to the fifth pair of leaves, and the phyllochron was estimated by the inverse of the slope of the regression between number of leaves and accumulated thermal sum. The number of leaves was counted every three days until the fifth pair of leaves was emitted on the main stem and the base temperature was estimated using the least variability method and by the combination of the relative development and linear regression methods. For the Q13-31 quinoa GENOTYPE, THE PHYLLOCHRON VALUE IS $28^{\circ} \mathrm{C}$ LEAF DAY- 1 WHILE THE BASE TEMPERATURE (TB) FOR LEAF EMISSION IS $7^{\circ} \mathrm{C}$. 
Munareto et al.

\section{INTRODUÇão}

Em cenários de mudanças climáticas que preveem aumentos de temperatura e períodos de seca, a produção de alimentos para uma população crescente, demanda pesquisas, a fim de, identificar culturas que sejam tolerantes a essas condições ambientais. A quinoa (Chenopodium quinoa Willd), um pseudocereal milenar, nativa da Região Andina é caracterizada por tolerar condições ambientais extremas como a seca, geada, salinidade do solo e alta radiação solar. O cultivo em diferentes ambientes, desde a Colômbia até o sul do Chile capacitou a planta a desenvolver características agronômicas e adaptativas específicas a essas regiões, variando desde o nível do mar até $4.000 \mathrm{~m}$ de altitude (GONZÁLEZ, 2011; CALLA, 2012).

A quinoa vem ganhando notoriedade no cenário mundial, não somente pela sua variabilidade genética e plasticidade fenotípica, mas, também pela qualidade nutricional dos seus grãos, vem sendo considerada uma cultura importante para melhorar a segurança alimentar mundial. Seus grãos que são fonte de proteína de alta qualidade, minerais, vitaminas, ácidos graxos poli-insaturados e aminoácidos essenciais a tornam um alimento quase completo (VEGA-GÁLVEZ, 2010; REPO-CARRASCO; SERNA, 2011).

A variedade de genótipos existentes, na Região Andina, tem permitido o cultivo fora desse ambiente, segundo a Organização para a Alimentação e Agricultura (FAO), mais de 95 países ao redor do mundo estão testando diferentes materiais genéticos de quinoa, com a finalidade de avaliar o manejo agrícola e o desempenho produtivo dessa espécie, identificando genótipos adaptados a cada região testada (BERTERO, 2001; PETERSON; MURPHY, 2015).

Dentre os aspectos pesquisado, a disponibilidade térmica de cada região de cultivo é o que mais tem influenciado no crescimento e no desenvolvimento das plantas. Desde modo, modelos são utilizados para determinar as necessidades térmicas das plantas pelo acúmulo térmico em graus-dia, considerando as temperaturas efetivas delimitadas pela temperatura basal inferior ( $\mathrm{Tb}$ ) e superior (TB). O limite inferior $\mathrm{Tb}$ corresponde à temperatura a partir da qual o metabolismo vegetal é acionado, e o limite superior TB corresponde à temperatura máxima de adaptação, fora desses limites térmicos as plantas paralisam seu metabolismo e passam a sofrer estresse por frio ou calor (BERGAMASCHI; BERGONCI, 2017).

Embora os modelos de desenvolvimento atendam os limites térmicos ( $\mathrm{Tb}$ e $\mathrm{TB}$ ), a maioria dos trabalhos leva em consideração apenas o limite inferior (Tb), independente da fase fenológico, pois a TB dificilmente é atingida a campo (MÜLLER, 2009). Existem vários métodos para estimar a Tb, sendo este parâmetro importante no zoneamento agroclimático otimizando a produção de várias culturas, por exemplo, triticale (× triticosecale Wittmack) (PEDRO JR., 2004), azevém (Lolium multiflorum Lam.) (MÜLLER, 2009), arroz (Oryza sativa L.) (LAGO, 2009), canola (Brassica napus L.) (LUZ, 2012) e oliveira (Olea europaea L.) (MARTINS, 2012).

Outro aspecto de desenvolvimento importante é o filocrono, considerada uma medida de desenvolvimento definido como o tempo térmico necessário para o aparecimento de folhas sucessivas na haste principal da planta, tendo como unidade de tempo a soma térmica $\left({ }^{\circ} \mathrm{C} \mathrm{dia}{ }^{-1}\right)$, que pode ser usada para avaliar como a planta responde às condições ambientais ou para prever como vai responder a elas. O filocrono é resultado da combinação de fatores genéticos e ambientais, que interagem para produzir folhas nas plantas (XUE, 2004; STRECK, 2005; PRŽULJ; MOMČILOVIĆ, 2013). Esses modelos de desenvolvimento tem sido amplamente aceito pelos pesquisadores nas culturas anuais, como: trigo (Triticum spp.) (ROSA, 2009), tomate (Solanum lycopersicum L.) (PIVETTA., 2007), milho (Zea mays L.) (STRECK, 2009), cevada (Hordeum vulgare L.) (PRŽULJ; MOMILOVI, 2013), entre outros.

O conhecimento das necessidades térmicas do desenvolvimento como, temperatura base e emissão de folhas, é determinante, pois auxiliam no planejamento agrícola, na escolha de cultivares mais adaptadas às condições climáticas de cultivo e na tomada de decisão quanto ao melhor momento para a execução de diferentes práticas agrícolas (irrigação, fertilização e tratamentos fitossanitários) melhorando a produção e o aproveitamento dos insumos (MONTEIRO, 2009; BERGAMASCHI; BERGONCI, 2017). Por isso, a 
tomada de decisão deve ser precisa, o que representa eficiência econômica e competitividade, dentro da atividade de risco como a agricultura. Com o propósito de aportar informações agronômicas pertinentes às exigências térmicas da cultura da quinoa, o objetivo deste trabalho foi estimar a temperatura base $(\mathrm{Tb})$ e determinar o filocrono do genótipo Q13-31.

\section{MATERIAL E MÉTODOS}

O experimento foi realizado na área do Departamento de Fitotecnia da Universidade Federal de Santa Maria, RS (2943' S, 5343’ W e altitude de 95 m). Pela classificação de Köppen, o clima pertence ao tipo Cfa, subtropical úmido, com verões quentes e sem estação seca definida. O solo da área classificado como Argissolo Vermelho Distrófico arênico (EMBRAPA, 2018).

O experimento no campo foi composto por um genótipo de quinoa e oito datas de semeadura, em três repetições, seguindo o delineamento de blocos ao acaso em parcelas de $2 \times 2 \mathrm{~m}$. O genótipo utilizado foi o Q1331, oriundo do Programa de Melhoramento de Quinoa da Universidade Estadual do Oeste do Paraná, PR.

As datas da semeadura foram 01/12/2016, 19/01/2017, 01/04/2017, 01/05/2017, 07/10/2017, 09/11/2017, $07 / 12 / 2017$ e 02/01/2018. A semeadura foi realizada de forma manual, em quatro fileiras de um metro, espaçadas a $0,5 \mathrm{~m}$ entre si com densidade de 40 plantas $\mathrm{m}^{2}$. As duas fileiras centrais de cada unidade experimental foram consideradas como área útil da parcela. O preparo do solo foi realizado de forma convencional, com duas gradagens. A partir da análise química do solo, foi realizada a adubação de base de $250 \mathrm{~kg} \mathrm{ha}^{-1}$, da fórmula 05-20-20, depositado a $5 \mathrm{~cm}$ abaixo das sementes, e estas de 1,0 a 2,0 $\mathrm{cm}$ de profundidade. A adubação de cobertura foi $150 \mathrm{~kg}$ de nitrogênio $(\mathrm{N})$ na forma de ureia, dividida em duas aplicações, sendo a primeira aos 20 dias após a emergência (DAE) e a segunda, no início da emissão da panícula. Foi realizado o controle das plantas daninhas (capina manual) doenças e pragas sempre que necessário.

A data da emergência (EME) foi considerada quando 50\% das plântulas estavam com cotilédones acima do nível do solo. Após a emergência foram marcadas, de forma aleatória, três plantas por parcela. Posteriormente, foram realizadas observações a cada três dias, registrando o número de folhas visíveis na haste principal, a partir da emergência até o quinto par de folhas da haste principal.

Para o cálculo da soma térmica acumulada (STa), acumulou-se a STd a partir da data de emergência, em cada época de semeadura, expressa na Equação 1.

$$
S T d=(\text { Tmed }-\mathrm{Tb})^{\circ} \mathrm{C} \text { dia }
$$

Sendo, o STd =0, quando $\mathrm{Tb}>$ tmed, adaptada pela Equação 2.

$$
\mathrm{STa}=\Sigma \mathrm{STd}^{\circ} \mathrm{C} \text { dia }
$$

Em que: STd é a soma térmica diária $\left({ }^{\circ} \mathrm{C}\right.$ dia $\left.^{-1}\right)$; Tmed é a temperatura média do ar $\left({ }^{\circ} \mathrm{C}\right)$ e $\mathrm{Tb}$ é a temperatura base $\left({ }^{\circ} \mathrm{C}\right)$. A temperatura base $(\mathrm{Tb})$ foi estimada pelos métodos da menor variabilidade propostas por Arnold (1959) e pela combinação dos métodos do desenvolvimento relativo e de regressão linear, proposto por Gbur (1979).

A soma térmica diária (STd) foi calculada com valores de $\mathrm{Tb}$ no intervalo de 0 a $15^{\circ} \mathrm{C}$, com incremento de $0,5^{\circ} \mathrm{C}$. Após calcular a soma térmica no referido intervalo escolhido, foi determinado o desvio-padrão em graus-dia (DPgg), para cada época de cultivo. Foi considerado como valor de Tb da cultura, aquela que correspondeu ao menor valor de desvio-padrão em dias (DPd), calculado a partir da Equação 3:

$$
\text { DPd=DPgd / (Tmed - Tb) }
$$


Em que: DPd é o desvio padrão em dias; Tmed é a temperatura média de todas as épocas de cultivo; o DPgd é o desvio padrão em graus-dias, para a série de épocas de semeadura, para cada valor de Tb.

O método do desenvolvimento relativo (DR) foi estimado a partir da Equação 4:

$$
\mathrm{DR}=\mathbf{a}+\mathbf{b} * \text { Tmed }
$$

Em que: Tmed é a temperatura média do ar $\left({ }^{\circ} \mathrm{C}\right)$ da série de datas de emergências; a e b são respectivamente os coeficientes linear e angular da regressão linear simples. O DR é calculado pela Equação 5:

$$
\mathrm{DR}=100 / \mathrm{n}
$$

Em que: DR a determinada temperatura média do ar $\left({ }^{\circ} \mathrm{C}\right) ; 100$ representa um valor arbitrário de ponderação; n são os dias decorridos da emergência até a emissão do quinto par de folhas. Quando DR for igual à zero, Tmed será igual a Tb; sendo obtida pela Equação 6:

$$
\mathbf{T b}=-\mathbf{a} / \mathbf{b}
$$

Posteriormente, realizou-se uma regressão linear simples entre número de folhas na haste principal e a soma térmica acumulada. A estimativa do filocrono foi realizada através do inverso do coeficiente angular (1/a), da regressão linear (XUE, 2004; STRECK, 2005).

\section{RESULTADOS E DISCUSSÃo}

As médias das temperaturas mínimas e máximas absolutas do ar durante a condução do experimento foram respectivamente, $16,3^{\circ} \mathrm{C}$ e $26,5^{\circ} \mathrm{C}$ (Figura 1). Esses valores de temperaturas ficaram dentro da faixa de 15 a $25^{\circ} \mathrm{C}$, considerada como ideal para o crescimento e desenvolvimento da cultura por Pando e Castellanos (2016).

Figura 1 - Normal climatológica e precipitação $(\mathrm{mm})$, e valores médios mensais de temperatura mínima do $\operatorname{ar}\left(\operatorname{Tmin},{ }^{\circ} \mathrm{C}\right)$ e temperatura máxima do $\operatorname{ar}\left(\operatorname{Tmax},{ }^{\circ} \mathrm{C}\right)$ correspondentes aos períodos de condução do experimento de quinoa (C. quinoa) de dezembro de 2016 a abril de 2018, Santa Maria, RS.

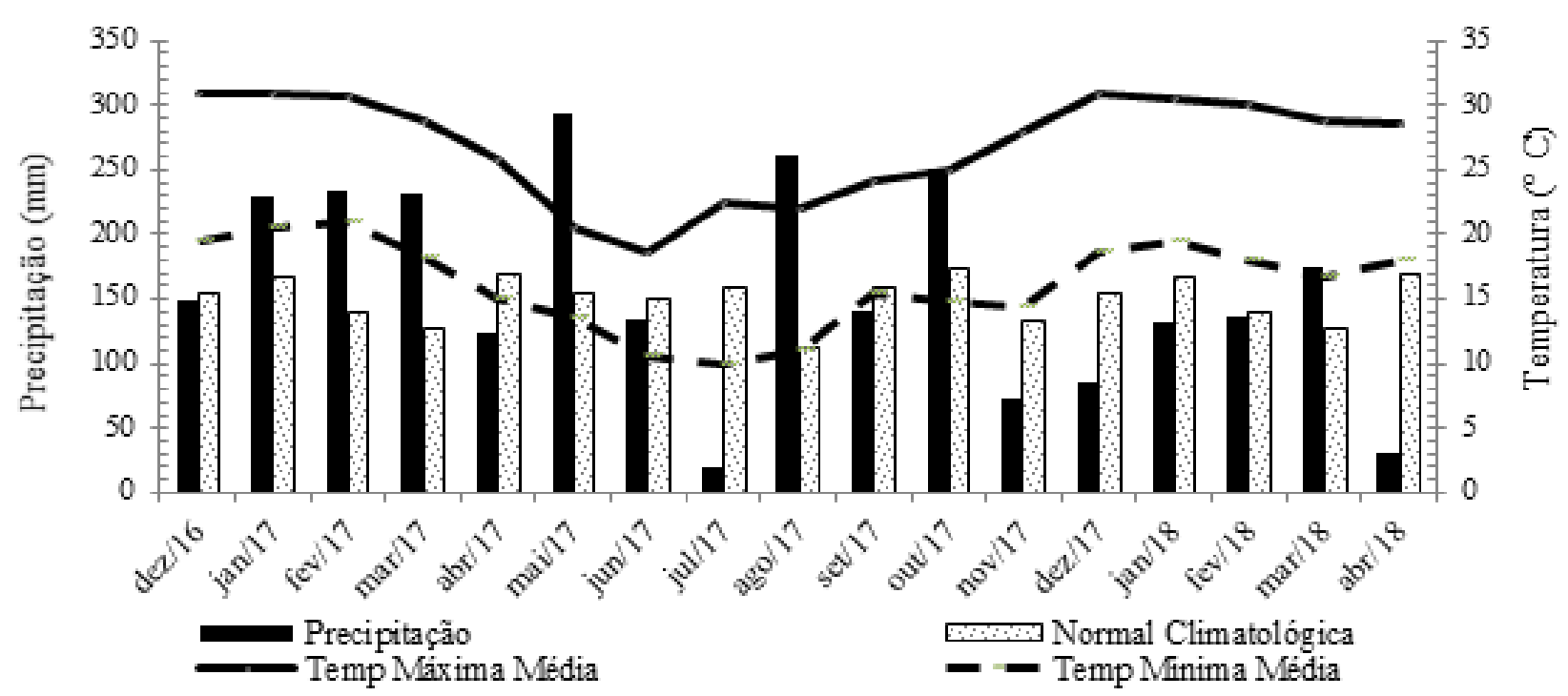

Fonte: elaborado pelo autores.

O total pluviométrico registrado no ano de 2017 foi de 2.080,6 mm (Figura 1), ficando acima da normal climatológica, pois Heldwein (2009) classificou média esperada para região de Santa Maria neste período 
de 1.712,4 mm. Os excessos hídricos ocorreram nos meses de janeiro $(62,9 \mathrm{~mm})$, fevereiro $(95 \mathrm{~mm})$, março $(104,7 \mathrm{~mm})$, maio $(139,6 \mathrm{~mm})$, agosto $(150,6 \mathrm{~mm})$ e outubro $(76,5 \mathrm{~mm})$ de 2017 . Durante esses meses foi observado alta incidência de fungos de solo (Rhizoctonia spp., Fusarium spp., Pythium spp.) causando morte de plantas, podridão do talo (Phoma spp.) e, um complexo de doenças foliares. Segundo Calla (2012), a planta de quinoa se desenvolve bem em ambientes secos com precipitações anuais entre 300 a $500 \mathrm{~mm}$, pois não tolera solos saturados de água.

As médias das temperaturas mínimas e máximas absolutas do ar durante a condução do experimento foram respectivamente, 16,3 e $26,5^{\circ} \mathrm{C}$ (Figura 1). Esses valores de temperaturas ficaram dentro da faixa de 15 a $25^{\circ} \mathrm{C}$, considerada como ideal para o crescimento e desenvolvimento da cultura (PANDO; CASTELLANOS, 2016).

Verificou-se que as estimativas dos valores de Tb variaram com os métodos utilizados, sendo que pelo valor de desvio-padrão em dias (DPd), houve a oscilação entre 5 e $7^{\circ} \mathrm{C}$, e pelo método do desenvolvimento relativo (DR) oscilou entre 6,8 a 9,5 $\mathrm{C}$ (Figura 2). Diferenças entre valores de $\mathrm{Tb}$, também foram observados por Müller et al. (2009), Luz (2012) e Souza e Martins (2014) em azevém, canola e oliveira, respectivamente, utilizando as mesmas metodologias. Para Andrade (2006) e Lago et al. (2009), as variações nos valores de $\mathrm{Tb}$ podem estar relacionadas à metodologia das estimativas, que desconsideram fatores ambientais importantes como temperatura radiação solar e fotoperíodo.

Em virtude da variabilidade nas Tb estimadas pelos dois modelos utilizados, assumiu-se a média dos valores obtidos, portanto considerando-se o valor de $\mathrm{Tb}$ igual a $7^{\circ} \mathrm{C}$. O limite da $\mathrm{Tb}$ de $7^{\circ} \mathrm{C}$ assumido para o genótipo Q13-31 é superior aos 3,7 e próximo a 6,4 $\mathrm{C}$ relatadas por Bertero (1996) para as variedades Amarilla de Maranganí (Peru) e Baer (Chile) respectivamente. Conforme observado cada genótipo possui uma $\mathrm{Tb}$, o que possibilita que a cultura se desenvolva em condições ambientais distintas. No Paraná, Vasconcelos et al. (2012) observaram que o desenvolvimento da cultivar BRS Piabiru não ocorreu abaixo de $7^{\circ} \mathrm{C}$, podendo ser semeada na entre safra das culturas principais (soja e milho) e alcançar produtividade média de $1.884 \mathrm{~kg} \mathrm{ha}^{-1}$. Melhorando o manejo agrícola e utilizando genótipos adaptados a Região Sul do país, a cultura pode alcançar o máximo potencial que é de $7.000 \mathrm{~kg} \mathrm{ha}^{-1}$ (PANDO; CASTELLANOS, 2016).

O número de folhas apresentou uma relação linear positiva com a soma térmica acumulada (Figura 3). O elevado coeficiente de determinação $\left(\mathrm{R}^{2}\right)$ de 0,92 demonstrou a forte associação entre a temperatura e a emissão de folhas, fato que também foi verificado por Pivetta (2007), Streck (2007) e Maldaner (2009) para as culturas de tomate, arroz e berinjela (Solanum melongena L.), respectivamente.

O filocrono de $28^{\circ} \mathrm{C}$ dia folha ${ }^{-1}$ encontrado para o genótipo Q13-31 (Figura 3), está próximo aos $22^{\circ} \mathrm{C}$ dia folha ${ }^{-1}$ obtidos por Bertero et al. (2003) em quinoas cultivadas em Clima tropical. Entretanto, valores inferiores, (12,9 e 17,2 ${ }^{\circ} \mathrm{C}$ dia folha ${ }^{-1}$ ), foram encontrados por Bois (2006). Para Bertero (2001), outros fatores podem influenciar o filocrono, inibindo parcialmente o efeito da temperatura do ar, sendo neste caso o fotoperíodo e a radiação solar,

Estudando os efeitos do fotoperíodo, da emergência ao início do período reprodutivo, no filocrono usando de fotoperíodos crescentes, Curti (2016) observaram que, à medida que, o fotoperíodo foi aumentado a duração do período vegetativo foi prolongado, respondendo quantitativamente a dias curtos. Como o cultivo das plantas ocorreu sob fotoperíodos crescente e temperaturas do ar elevadas (Figura 1), fato que pode ter antecipado a indução à floração, reduzindo a taxa de emissão de folhas e como consequência aumentou o filocrono.

Em trigo (ROSA, 2009) e canola (DALMAGO, 2013), relatam que o filocrono variou entre cultivares e épocas de semeadura, e acrescentam que quando a semeadura ocorreu dentro do zoneamento agroclimático, os genótipos tardios apresentaram maior valor de filocrono que os precoces.

Figura 2 - Determinação da temperatura base inferior $\left(\mathrm{Tb} ;{ }^{\circ} \mathrm{C}\right)$ para o subperíodo germinação a emissão do primeiro (A), segundo (B), terceiro (C), quarto (D) e quinto (E) par de folhas pelo método do menor 


\section{Munareto et al.}

valor de desvio-padrão em dias (DPd) e pelo método de desenvolvimento relativo (DR) para o genótipo de quinoa (C. quinoa) Q13-31 em Santa Maria, RS.
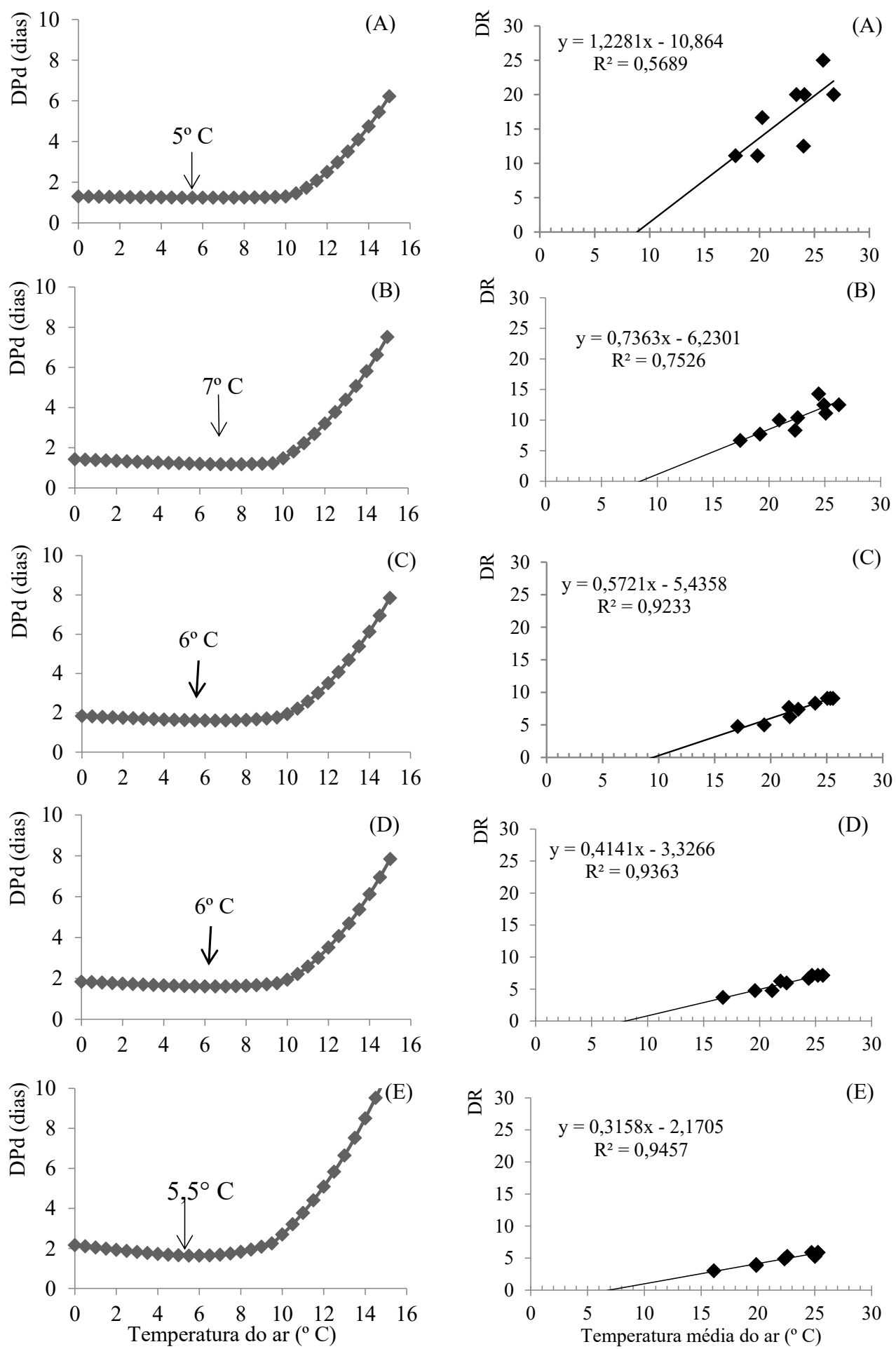

Valor de desvio-padrão em dias (DPd) Método de desenvolvimento relativo (DR). 
Figura 3 - Relação entre o número de folhas (NF) e a soma térmica acumulada (STa) utilizada para estimar o filocrono do genótipo (C. quinoa) Q13-31. Santa Maria, RS.

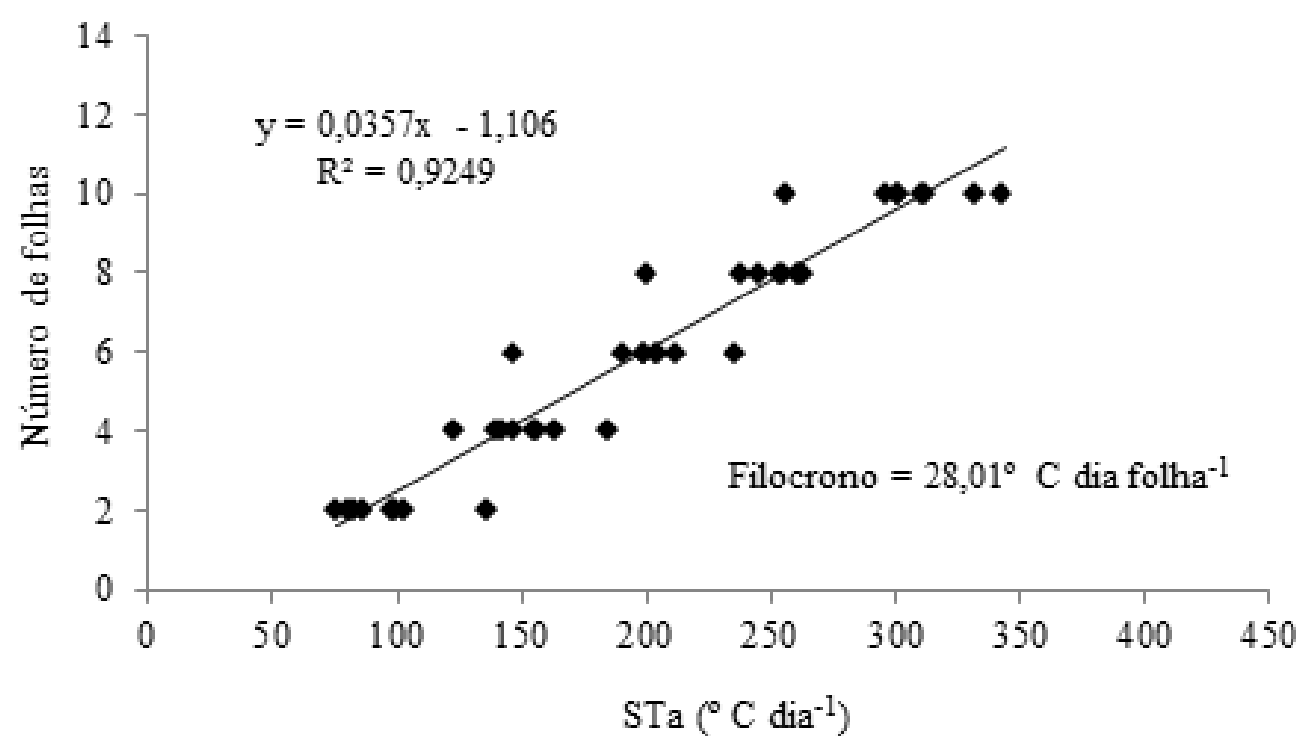

Fonte: elaborado pelo autores.

\section{Conclusão}

O desenvolvimento da cultura de quinoa (genótipo Q13-31) é viável na região de Santa Maria, RS. Tendo o valor do filocrono é $28^{\circ} \mathrm{C}$ dia folha ${ }^{-1}$, enquanto que a temperatura base (Tb) para a emissão de folhas é de $7^{\circ} \mathrm{C}$.

\section{REFERÊNCIAS}

ANDRADE, R.G; SEDIYAMA, G.C.; ZOLNIER, S.; COSTA, L.C. Avaliação dos modelos beta, rcm e GDD para diferentes subperíodos Linear Heat Unit System. American Society for Horticulture Science, v. 74, n. 1, p. $430-445,1959$.

ARNOLD, C. Y. The Determination and Significance of the Base Temperature in a Linear Heat Unit System. American Society for Horticulture Science, v. 74, n. 1, p. 430-445, 1959.

BERGAMASCHI, H.; BERGONCI, J.I. Temperatura do ar em As Plantas e o Clima - Princípios e aplicações. Guaíba: Agrolivros, 2017.351p.

BERTERO, D.; MEDAN, D.; HALL, A. J. Changes in apical morphology during floral initiation and reproductive development in quinoa (Chenopodium quinoa Willd.). Annals of Botany, v.78, n. 2, p.317-324, 1996. BERTERO, H. D. Response of developmental processes to temperature and photoperiod in quinoa (Chenopodium quinoa Willd). Food Reviews Intemational. v. 19, n. 1, p. 87-97, 2003.

BERTERO, H. D. Effects of photoperiod, temperature and radiation on the rate of leaf appearance in quinoa (Chenopodium quinoa Willd.) under field conditions. Annals of Botany, v. 87, n. 1, p.495-502, 2001. 
Munareto et al.

BOIS, J. F.; WINKEL, T.; LHOMME, J.P.; RAFFAILLAC, J.P.; ROCHETEAU, A. Response of some Andean cultivars of quinoa (Chenopodium quinoa Willd.) to temperature: Effects on germination, phenology, growth and freezing. European Journal of agronomy, v. 25, n. 3, p. 299-308, 2006.

CALLA, J. MANEJO. AGRONÓMICO DEL CULTIVO DE LA QUINUA. PERÚ: AGROBANCO. GUÍA TÉCNICA. 2012, 40 P.

CURTI, R. N.; DE LA VEGA, A. J.; ANDRADE, A. J.; BRAMARDI, S. J.; BERTERO, H. D. Adaptive responses of quinoa to diverse agro-ecological environments along an altitudinal gradient in North West Argentina. Field Crops Research, v. 189, n. 1, p. 10-18, 2016.

DALMAGO, G.; CUNHA, G.R.; SANTI, A.; PIRES, J.L.F.; MÜLLER, A.L.; BOLIS, L.M. Filocrono e número de folhas da canola em diferentes condições ambientais. Pesquisa Agropecuária Brasileira, v. 48 , n. 1, p. 573-581, 2013.

EMBRAPA - Empresa Brasileira de Pesquisa Agropecuária. Sistema Brasileiro de Classificação de Solos. 5. ed., Brasília, DF, 2018. 156 p.

GBUR, E. E.; THOMAS; G. L.; MILLER, F. R. Use of segmented regression in determination of the base-temperature in heat accumulation models. Agronomy Journal, v. 71, n. 2, p. 949-953, 1979.

GONZÁLEZ, J. A.; AGUILAR, P. C.; S. E. JACOBSEN. Genotypic variation of gas exchange parameters and leaf stable carbon and nitrogen isotopes in ten quinoa cultivars grown under drought. Journal of Agronomy and Crop Science, v. 197, n. 1, p.81-93, 2011.

HELDWEIN, A. B.; BURIOL, G. A.; STRECK, N. A. O clima de Santa Maria. Ciência \& Ambiente, v. 38, n. 4, p. $43-58,2009$.

LAGO, I.; STRECK, N. A.; DE CARVALHO, M. P.; FAGUNDES, L. K.; DE PAULA, G. M.; LOPES, S. J. Estimativa da temperatura base do subperíodo emergência-diferenciação da panícula em arroz cultivado e arroz vermelho. Revista Ceres, v. 56, n. 3, p. 288-295, 2009.

LUZ, G. L. D.; MEDEIROS, S. L. P.; TOMM, G. O.; BIALOZOR, A.; AMARAL, A. D. D.; PIVOTO, D. Temperatura base inferior e ciclo de híbridos de canola. Ciência Rural, v. 42, n. 9, p. 1549-1555, 2012.

MALDANER, I. C.; GUSE, F. I.; STRECK, N. A.; HELDWEIN, A. B.; LUCAS, D. D. P.; LOOSE, L. H. Filocrono, área foliar e produtividade de frutos de berinjela conduzidas com uma e duas hastes por planta em estufa plástica. Ciência Rural, v. 39, n. 1, p. 671-677, 2009.

MARTINS, F. B.; REIS, D. D. F.; PINHEIRO, M. V. M. Temperatura base e filocrono em duas cultivares de oliveira. Ciência Rural, v. 2, n. 1, p.1975-1981, 2012.

MONTEIRO, J. E. B. A agrometeorologia dos cultivos. O fator meteorológico na produção agrícola. Brasília, DF: INMET, 2009. 530 p.

MÜLlER, L.; MANFRON, P. A.; MEDEIROS, S. L. P.; STRECK, N. A.; MITTELMMAN, A.; DOURA- 
DO NETO, D.; MORAIS, K. P. Temperatura base inferior e estacionalidade de produção de genótipos diploides e tetraploides de azevém. Ciência Rural, v. 39, n. 5, p.1343-1348, 2009.

PANDO, L. G.; CASTELLANOS, E. A. Guia de cultivo de la quinua. FAO. Organización de las Naciones Unidas para la Alimentación y la Agricultura e Universidad Nacional Agraria La Molina, 2016. Disponível em: <www.fao.org/3/a-i5374s.pdf>. Acesso em: 14 set. 2019.

PEDRO JÚNIOR, M. J.; CAMARGO, M. B. P. D.; MORAES, A. V. D. C.; FELÍCIO, J. C.; CASTRO, J. L. D. Temperatura base, graus-dia e duração do ciclo para cultivares de triticale. Bragantia, Campinas, v.63, n.3, p.447-453, 2004.

PETERSON, A.; MURPHY, K. M. Quinoa cultivation for temperate North America: considerations and areas for investigation. In: MURPHY, K. M.; MATANGUIHAN, J. G. Quinoa: Improvement and Sustainable Production. Hoboken: John Wiley \& Sons, 2015. p.173-192.

PIVETTA, C. R.; TAZZO, I. F.; MAASS, G. F.; STRECK, N. A.; HELDWEIN, A. B. Emissão e expansão foliar em três genótipos de tomateiro (Lycopersicon esculentum Mill.). Ciência Rural, v. 37, n. 1, p.12741280, 2007.

PRŽULJ, N.M.; MOMČILOVIĆ, V.M. Effect of cultivar and year on phyllochron in winter barley. Journal of Natural Sciences, v. 125, n. 1, p. 93-100, 2013.

REPO-CARRASCO-VALENCIA, R. A. M.; SERNA, L. A. Quinoa (Chenopodium quinoa, Willd.) as a source of dietary fiber and other functional components. Ciência e Tecnologia de Alimentos, v. 31, n. 1, p. 225-230, 2011.

ROSA, H. T.; WALTER, L. C.;STRECK, N. A.; ALBERTO, C. M. Filocrono de trigo em função de métodos de soma térmica. Pesquisa Agropecuária. Brasileira. v. 44, n. 2, p.1374-1382, 2009.

SOUZA, P. M. B.; MARTINS, F. B. Estimativa da temperatura basal inferior para as cultivares de oliveira Grappolo e Maria da Fé. Revista Brasileira de Meteorologia, v. 29, n. 1, p. 307-313, 2014.

STRECK, N. A.; BOSCO, L. C.; MENEZES, N. L.; GARCIA, D. C.; ALBERTO, C. M.; LAGO, I. Estimativa do filocrono em cultivares de trigo de primavera. Revista Brasileira de Agrometeorologia, v. 13, n. 1, p.423-429, 2005.

STRECK, N. A.; MICHELON, S.; ROSA, H. T.; WALTER, L. C.; BOSCO, L. C.; PAULA, G. M. D.; LOPES, S. J. Filocrono de genótipos de arroz irrigado em função da época de semeadura. Ciência Rural, v. 37, n. 2, p.323-329, 2007.

STRECK, N. A.; LAGO, I.; SAMBORANHA, F. K.; GABRIEL, L. F.; SCHWANTES, A. P.; SCHONS, A. Temperatura base para aparecimento de folhas e filocrono da variedade de milho BRS Missões. Ciência Rural, v. 39, n. 1, p.224-227, 2009.

VASCONCELOS, F. S. Desenvolvimento e produtividade de quinoa semeada em diferentes datas no período safrinha. Revista Ciência agronômica, v. 43, n. 1, p. 510-515, 2012. 


\section{Munareto et al.}

VEGA-GÁlVEZ, A.; MIRANDA, M.; VERGARA, J.; URIBE, E.; PUENTE, L.; MARTÍNEZ, E. A. Nutrition facts and functional potential of quinoa (Chenopodium quinoa Willd.), an ancient Andean grain: a review. Journal of the Science of Food and Agriculture, v. 90, n. 1, p. 2541-2547, 2010.

XUE, Q.; WEISS, A.; BAENZIGER, P.S. Predicting leaf appearance in field-grown winter wheat: evaluating linear and non-linear models. Ecological Modelling, v. 175, n. 1, p. 261-270, 2004. 